\title{
Significance of imaging and clinical features in the differentiation between primary and secondary pulmonary lymphoma
}

\author{
YUHAO DONG ${ }^{1,2^{*}}$, MIAOYU ZENG ${ }^{3 *}$, BIN ZHANG $^{1,3}$, LUJUN HAN $^{4}$, ENTAO LIU ${ }^{5}$, \\ ZHOUYANG LIAN $^{1,3}$, JING LIU $^{1,3}$, CHANGHONG LIANG ${ }^{1}$ and SHUIXING ZHANG ${ }^{1}$ \\ ${ }^{1}$ Department of Radiology, Guangdong Academy of Medical Sciences/Guangdong General Hospital, Guangzhou, \\ Guangdong 510080; ${ }^{2}$ Graduate College, Shantou University Medical College, Shantou, Guangdong 515041; \\ ${ }^{3}$ Graduate College, Southern Medical University; ${ }^{4}$ Department of Radiology, Sun Yat-sen University Cancer \\ Center, Guangzhou, Guangdong 510120; ${ }^{5}$ Department of Nuclear Medicine, Guangdong Academy of \\ Medical Sciences/Guangdong General Hospital, Guangzhou, Guangdong 510080, P.R. China
}

Received August 18,2016; Accepted July 11, 2017

DOI: $10.3892 / 01.2017 .6962$

\begin{abstract}
The aim of the present study was to analyze the differences in imaging and clinical features between primary pulmonary lymphoma (PPL) and secondary pulmonary lymphoma (SPL) to provide insight into pulmonary lymphoma for an improved clinical diagnosis. A retrospective study of 38 patients with pulmonary lymphoma (19 PPL and 19 SPL) treated between September 1, 2006, and December 31, 2015, was performed. The clinical manifestations, and computed tomography (CT) and positron emission tomography-CT images of each case were collected. $\chi^{2}$ and Fisher's exact tests were applied to assess statistically significant differences between PPL and SPL in terms of clinical and imaging features. The significant variables were further applied to canonical discriminate analysis. The CT results revealed that the occurrence of a $>3-\mathrm{cm}$ mass $(\mathrm{P}=0.007)$, peripheral location (lower than the segmental bronchi) $(\mathrm{P}=0.027)$, cavitation $(\mathrm{P}=0.008)$ and consolidation $(\mathrm{P}=0.027)$ were associated with $\mathrm{PPL}$, while peripheral and hilar location $(\mathrm{P}=0.003)$ or mediastinal and
\end{abstract}

Correspondence to: Dr Shuixing Zhang, Department of Radiology, Guangdong Academy of Medical Sciences/Guangdong General Hospital, 106 Zhongshan Er Road, Guangzhou, Guangdong 510080, P.R. China

E-mail: shui7515@126.com

${ }^{*}$ Contributed equally

Abbreviations: PPL, primary pulmonary lymphoma; SPL, secondary pulmonary lymphoma; MALT, mucosa-associated lymphoid tissue; BALT, bronchial mucosa-associated lymphoid tissue; DLBCL, diffuse large B cell lymphoma; NCCN, National Comprehensive Cancer Network; $\mathrm{SUV}_{\max }$, maximum standard uptake value; NK/T, natural killer/T cell

Key words: primary pulmonary lymphoma, secondary pulmonary lymphoma, computed tomography, clinical features, diagnosis, discriminate analysis hilar lymph node engagement $(\mathrm{P}=0.044)$ were predominantly observed in SPL. However, no significant differences between clinical manifestations and the maximum standard uptake value of pulmonary lesions in PPL and SPL were identified (all $\mathrm{P}>0.05)$. A function derived from discriminate analysis was generated that may predict the affiliation to PPL or SPL radiographically, with an overall accuracy of $\leq 92.1 \%$. The results of the present study revealed that PPL and SPL exhibit distinctive features on CT images due to distinct molecular mechanisms and growth patterns. Careful observation of CT features may be useful in the diagnosis of PPL and SPL regarding the tumor morphology, location and lymph node involvement.

\section{Introduction}

Pulmonary lymphomas may occur as a primary pulmonary malignancy or a secondary manifestation derived from systemic lymphoma (1). Primary pulmonary lymphomas (PPLs) are defined as malignant lymphomas originating from pulmonary parenchyma or bronchi with or without hilar lymph node involvement, in the absence of tumor dissemination for $\geq 3$ months after the diagnosis $(2,3)$. Prevalence ranges between 0.5 and $1 \%$ of PPL malignancies and accounts for $<1 \%$ of malignant lymphomas (4). The rarity of PPL may be attributed to pulmonary tissue containing less lymphoid tissue compared with other sites. The most common type of PPL is mucosa-associated lymphoid tissue (MALT) lymphoma (70-90\%), with the remainder comprising a miscellaneous group that includes non-Hodgkin low-grade extra-nodal B-cell lymphoma (NHL-B), high-grade diffuse large B-cell lymphoma (DLBCL) (5-20\%), primary pulmonary Hodgkin's lymphoma and certain other extremely rare diseases (5-7). Secondary pulmonary lymphoma (SPL), which is more common than PPL, refers to a secondary involvement of the lung from a known extra-pulmonary lymphoma or dominant pulmonary lesion, with indolent primary extra-pulmonary lesions observed within 3 months (8).

These two origins of pulmonary lymphoma distinguish themselves pathologically, but may share the same mediastinal 
manifestations with variant imaging features $(8,9)$. Standard treatment protocols for PPL remain under investigation and are typically based on expert opinion rather than evidence-based, while therapy for SPL varies from its primary lesions (2). Surgery has been suggested as the preferred treatment in cases of localized PPL, when complete resection may be achieved. Patients with SPL are typically treated with chemotherapy (10). Additionally, PPL, considering MALT as the most common type, exhibits with a better outcome compared with SPL (11). Chest, abdominal and pelvic enhanced-computed tomography (CT) are referred to as a basic diagnostic investigation of pulmonary lymphomas in the National Comprehensive Cancer Network (NCCN) guideline of 2015, while positron emission tomography (PET)-CT is recommended for in depth evaluation $(12,13)$. PET/CT possesses increased accuracy in the overall staging of lymphoma compared with $\mathrm{CT}$, and is often used alongside CT scans in the evaluation of patients with lymphoma (13).

The World Health Organization published a classification scheme in 2008 that grouped lymphomas by cell type, as well as by molecular, cytogenetic and phenotypic characteristics (14). Previous studies have demonstrated the radiological presentations of distinct pathological types of pulmonary lymphoma, as well as the differentiation between pulmonary carcinoma and other lymphoproliferative changes of the lung, and a consensus has been reached to a certain extent $(15,16)$. However, only a limited number of studies have investigated the differences between PPL and SPL, with conflicting results having been reported $(9,17,18)$. To the best of our knowledge, in only one investigation has the distinction in clinical manifestations and radiological features between PPL and SPL been taken into consideration (19). At present, the treatments are similar between PPL and SPL.

The aim of the present study was to analyze the imaging features and clinical manifestations of PPL and SPL to improve our understanding of their pathogenesis and growth patterns. The differences observed between PPL and SPL features in CT results may improve early detection, offer proper referral for further examinations and provide timely treatment for an improved prognosis.

\section{Patients and methods}

Eligibility. A total of 47 patients with pulmonary lymphoma were retrospectively identified through a key word search (pulmonary lymphoma) of pathological databases at Guangdong General Hospital (Guangzhou, China) who were treated between September 1, 2006, and December 31, 2015. A single patient with HIV infection was excluded from further analysis, as they presented with acquired immunodeficiency syndrome-associated lymphoma. A further 8 cases were excluded due to a lack of sufficient recorded details. Subsequently, 38 confirmed cases with thorough medical records were included in the present study, equally divided $(n=19)$ between PPL and SPL. The study group consisted of 16 women (42\%) and 22 men (58\%), with a mean patient age of 39 years (range, 21-77 years) and a male:female ratio of $1: 1.38$. The present study was ethically approved by the Institutional Ethics Committee of Guangdong General Hospital (Guangzhou, China).
Imaging protocol. All patients were scanned with a 64-slice CT scanner (GE Light Speed VCT scanner; GE Healthcare, Chicago, IL, USA). Imaging parameters were as follows: $230 \mathrm{mAsec} ; 120 \mathrm{kVp}$; field of view, 33x33 cm; slice thickness, $1.25-\mathrm{mm}$; and intersection gap, $1.25 \mathrm{~mm}$. Precontrast and enhanced images were obtained from the apex to the diaphragm. Extrathoracic involvement was excluded or confirmed using a standard abdominal CT scan and bilateral iliac crest bone marrow biopsies. A total of 15 patients had undergone fluorine-18 2-deoxy-2-fluoro-D-glucose (18F-FDG) PET scanning. PET/CT scans were performed on an RDS111 cyclotron scanner (Crystal Technology \& Industries, Inc., Addison, TX, USA). Patients were fasted for $\geq 6 \mathrm{~h}$ prior to scanning. Blood glucose was measured and recorded on the radiology information system prior to an intravenous injection of $4 \mathrm{MBq} / \mathrm{kg} 18 \mathrm{~F}-\mathrm{FDG}$. Imaging commenced $\sim 90 \mathrm{~min}$ after injection and included the area from the skull base to the upper thighs. The PET/CT images were captured under normal tidal respiration for $2 \mathrm{~min} / \mathrm{bed}$ position, for a total of 8-10 bed positions. The CT images were captured using the following parameters: Tube voltage, $120 \mathrm{kV}$; tube current, 100-500 mA; slice thickness, 5-mm; and slice interval, $5 \mathrm{~mm}$. Images were reconstructed with a $1.25-\mathrm{mm}$ slice thickness and 1.25-mm slice interval. CT scanning was performed prior to PET scanning. The maximum standard uptake value $\left(\mathrm{SUV}_{\max }\right)$ was recorded using a standard volume of interest tool (Wizard workstation 16; Siemans AG, Munich, Germany).

Two radiologists, who had specialized in thoracic diseases for $>10$ years, reviewed all cases independently for radiological evidence at initial diagnosis, the presenting signs and symptoms at the first visit, and previous attempted diagnostic procedures and therapeutic protocols. Histological specimens were reviewed to validate the diagnosis of lymphoma. Paraffin-embedded biopsy specimens (thickness, 2-3 $\mu \mathrm{m}$ ) were stained with hematoxylin and eosin for $10 \mathrm{~min}$ at room temperature, and subsequently observed under an Olympus light microscope (magnification, $\mathrm{x} 400$ ) Discrepancies in interpretation were solved by discussion or evaluated by a third radiologist.

Statistical analysis. $\chi^{2}$ or Fisher's exact tests were used to assess the statistical significance between PPL and SPL in terms of clinical and imaging features. Fisher's exact test is a widely used alternative to $\chi^{2}$ for comparing pairs of proportions in a $2 \times 2$ contingency table with small samples. All statistical tests were 2 -sided and $\mathrm{P}<0.05$ was considered to indicate a statistically significant difference. Significant variables associated with CT features were then applied in a canonical discriminant analysis to differentiate between the diagnosis of PPL and SPL. Prediction equation L was built based on significant variables and probability belonging to PPL, $1 /\left(1+\mathrm{e}^{-\mathrm{L}}\right)$.

\section{Results}

Patients. All patients were histologically confirmed with pulmonary lymphoma. A total of 12 patients underwent diagnostic video-assisted thoracic surgery, 7 underwent a lobectomy, 6 underwent a bronchoscope biopsy procedure and 13 underwent a CT-guided transthoracic needle biopsy procedure. A total of 19 patients were diagnosed with PPL ( 9 women 
Table I. Symptoms of patients upon diagnosis of PPL and SPL.

\begin{tabular}{|c|c|c|c|c|c|}
\hline \multirow[b]{2}{*}{ Clinical manifestations } & \multicolumn{2}{|c|}{$\operatorname{PPL}(n=19)$} & \multirow[b]{2}{*}{ P-value ${ }^{a}$} & \multirow[b]{2}{*}{$\operatorname{SPL}(n=19)$} & \multirow[b]{2}{*}{$\mathrm{P}$-value ${ }^{\mathrm{b}}$} \\
\hline & $\begin{array}{l}\text { MALT } \\
(n=10)\end{array}$ & $\begin{array}{l}\text { Non-MALT } \\
\quad(n=9)\end{array}$ & & & \\
\hline \multicolumn{6}{|l|}{ Pulmonary symptoms } \\
\hline Chest pain & 1 & 5 & 0.057 & 4 & 0.714 \\
\hline Dyspnea & 1 & 3 & 0.303 & 1 & 0.340 \\
\hline Hemoptysis & 0 & 0 & - & 1 & $>0.990$ \\
\hline Cough & 6 & 4 & 0.656 & 5 & 0.097 \\
\hline Chest tightness & 1 & 2 & 0.582 & 2 & $>0.990$ \\
\hline \multicolumn{6}{|l|}{ Systemic symptoms } \\
\hline Fever & 0 & 5 & $0.011^{\mathrm{c}}$ & 3 & 0.693 \\
\hline Fatigue & 1 & 1 & $>0.990$ & 3 & $>0.990$ \\
\hline Weight loss & 1 & 0 & $>0.990$ & 6 & 0.090 \\
\hline Night sweat & 1 & 0 & $>0.990$ & 1 & $>0.990$ \\
\hline Sore throat & 0 & 0 & - & 1 & $>0.990$ \\
\hline$>$ Stage I-E & 4 & 7 & 0.170 & 17 & $0.027^{\mathrm{c}}$ \\
\hline Asymptomatic & 3 & 1 & 0.582 & 2 & 0.660 \\
\hline
\end{tabular}

${ }^{a}$ MALT vs. non-MALT. ${ }^{b P P L}$ vs. SPL. ${ }^{\mathrm{c}} \mathrm{P}<0.05$. MALT, mucosa-associated lymphoid tissue; PPL, primary pulmonary lymphoma; SPL, secondary pulmonary lymphoma.

and 10 men; female:male ratio, $1: 1.1$; mean age \pm standard deviation, 46.7 \pm 3.5 years; range, 21-71 years), while 19 were diagnosed with SPL ( 7 women and 12 men; female:male ratio, 1:1.7; mean age \pm standard deviation, $44.9 \pm 4.2$ years; range, 22-77 years). Of the 19 patients with PPL, 10 cases were regarded as MALT NHL (5 women and 5 men; female:male ratio, $1: 1$; mean age \pm standard deviation, $49 \pm 5.2$ years; range, 39-67 years), 2 were DLBCL, 2 were T cell lymphoma and 1 was natural killer/T cell (NK/T) lymphoma, with the remaining 4 cases being diagnosed as classic Hodgkin's lymphoma, nodular sclerosis type (Fig. 1). For the 19 cases of SPL, 7 were diagnosed as DLBCL of NHL, 4 were T cell lymphoma of NHL, 3 were NK/T lymphoma of NHL and 1 was mantle cell lymphoma. classic Hodgkin's lymphoma, nodular sclerosis type, accounted for the 4 remaining cases (Fig. 1).

Clinical manifestations. The initial presenting symptoms of PPL and SPL are summarized in Table I. The predominant symptoms experienced by patients with PPL were coughing (53\%), chest pain (32\%), dyspnea (21\%) and fever (26\%), while $21 \%$ were asymptomatic compared with $11 \%$ asymptomatic patients with SPL. As the most common and well-characterized type of PPL, MALT lymphoma was observed and analyzed separately. However, no significant differences between the clinical manifestations of MALT and non-MALT lymphoma of NHL in PPL were identified, with the exception of fever $(\mathrm{P}=0.011)$. For SPL, 53\% of patients possessed intrathoracic or jugular lesions of indolent manifestation, as observed using CT. A single patient presented with inguinal lymphadenopathy, 1 with skin lesions, 4 with gastrointestinal lymphoma, 1 with splenic lymphadenopathy and 1 with nasopharyngeal lymphoma. In total, $\leq 32 \%$ of the patients complained of
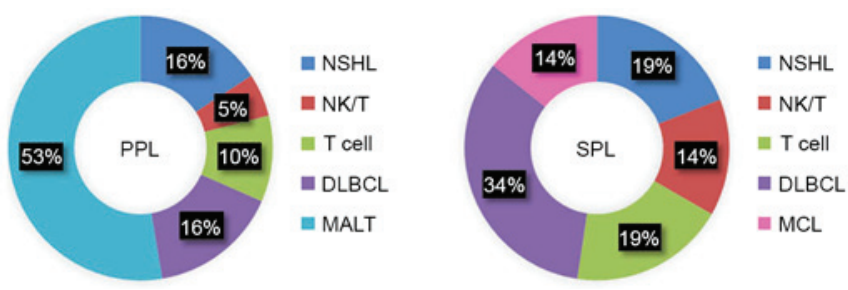

Figure 1. Histological types of PPL and SPL in the present study. PPL, primary pulmonary lymphoma; SPL, secondary pulmonary lymphoma; MALT, mucosa-associated lymphoid tissue; MCL, mantle cell lymphoma; DLBCL, diffuse large B cell lymphoma; NSHL, nodular sclerosis Hodgkin's lymphoma; NK/T, natural killer/T cell.

weight loss and 20-26\% experienced respiratory symptoms such as coughing and chest pain. Hemoptysis was rare and only observed in 1 patient. No statistically significant differences in respiratory or systemic symptoms between PPL and SPL were identified, with the exception of stage I-E $(\mathrm{P}=0.027)$.

\section{Imaging features}

CT. A comparison of the CT features of PPL and SPL is presented in Figs. 2-4. PPL was located more peripherally (lower than the segmental bronchi; $\mathrm{n}=8 ; \mathrm{P}<0.027$ ), while SPL disseminated in a more diffuse pattern with peripheral and hilar lesions $(n=14 ; \mathrm{P}<0.003)$. MALT $(n=7)$ were higher in number compared with the non-MALT of PPL $(n=1$; $\mathrm{P}<0.02)$. Cavitation occurred in $40 \%$ of the PPL group, while not being observed in patients with SPL. The most common CT abnormality in the PPL group was a mass or mass-like consolidation of homo-intense or marked heterogeneous 


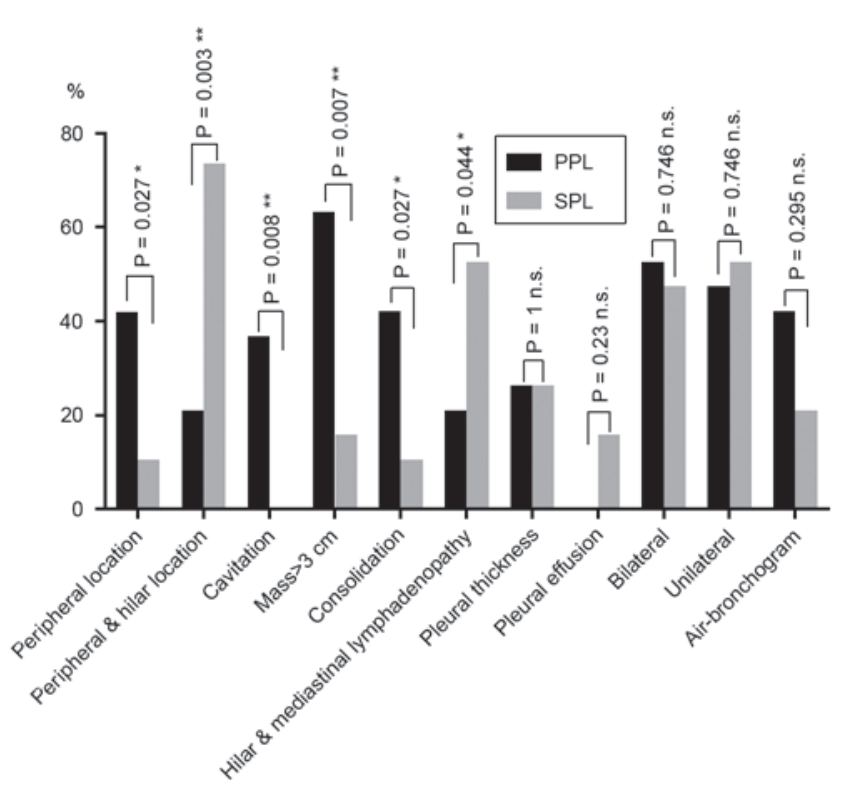

Figure 2. Comparison of computed tomography features in patients with PPL $(\mathrm{n}=19)$ or SPL $(\mathrm{n}=19)$. Note that more than one feature may be observed at one time. Significant differences were determined by $\chi^{2}$ and Fisher's exact test. ${ }^{*} \mathrm{P}<0.05,{ }^{* *} \mathrm{P}<0.01$ and ${ }^{* * * *} \mathrm{P}<0.001$. PPL, primary pulmonary lymphoma; SPL, secondary pulmonary lymphoma; n.s., not significant.

enhancement, observed in $12 / 19$ patients $(\mathrm{P}=0.007)$. The same was not true for SPL, in which the most common CT feature observed was hilar and mediastinal lymphadenopathy $(n=10$; $\mathrm{P}=0.044)$. Consolidation was observed more in the PPL group $(n=8)$ than the SPL group $(n=2 ; P=0.027)$. Of the 38 patients in the present study, 20 (53\%) experienced abnormalities in $\geq 2$ of the 6 categories in which significant differences were identified (Fig. 2).

As for the non-significant CT features, CT-angiogram and air-bronchogram, as well as pleural involvement in lymphoma are the most common features that cause debate among radiologists. Analysis of the cases in the present study revealed that the CT-angiogram sign (enhancing vessels observed within the mass or consolidation) was observed in $26 \%$ of the PPL and $11 \%$ of the SPL cases. Air bronchograms were observed in 8 PPL cases and 4 SPL cases, without significant differences. A total of 5 patients with PPL presented with pleural involvement, including pleural thickness or pleural effusion, while 8 cases were observed in the SPL group. Vessel engagement was observed in 5 cases of PPL and 2 cases of SPL, without significant differences. With regard to lobular involvement, the present study observed that PPL and SPL were able to manifest as bilateral or unilateral lobular involvement, without preferential significance.

PET-CT. A total of 15 patients underwent PET-CT scanning, including 8 in the PPL group and 7 in the SPL group. A total of $6 / 8$ patients with PPL were MALT type, presenting with a unilateral or bilateral nodule or mass $>3 \mathrm{~cm}$, and the remaining 2 were validated with classic Hodgkin's lymphoma. $\mathrm{SUV}_{\max }$ of the primary site for these 8 cases ranged between 2.3 and 11.3, with 3.8 as the median and $6.0( \pm 3.7)$ as the mean value. The extrapulmonary lymphadenopathy of SPL in the present study involved hilar, mediastinal, neck, stomach, small intestine
Table II. Classification for PPL and SPL by means of radiographic features.

\begin{tabular}{lccc}
\hline & \multicolumn{2}{c}{$\begin{array}{c}\text { Predicted diagnosis based } \\
\text { on radiographic features }\end{array}$} \\
\cline { 2 - 4 } $\begin{array}{l}\text { Actual diagnosis based on } \\
\text { histological test }\end{array}$ & PPL & SPL & Total \\
\hline PPL & 19 & 0 & 19 \\
SPL & 3 & 16 & 19 \\
Total & 22 & 16 & 38 \\
\hline
\end{tabular}

PPL, primary pulmonary lymphoma; SPL, secondary pulmonary lymphoma.

and pelvic regions, among others. The range of $\mathrm{SUV}_{\max }$ in these lesions was between 6.6 and 10.9. The $\mathrm{SUV}_{\max }$ of the pulmonary lesions ranged between 1.3 and 18.4 , with 3.5 as the median and $7.8( \pm 7.0)$ as the mean value.

Differential analysis. No statistically significant differences for $\mathrm{SUV}_{\max }$ PET/CT between PPL and SPL were identified. However, the statistical analysis of the $\mathrm{CT}$ results revealed 6 features that may assist in the radiological differentiation between PPL and SPL (Fig. 2). The major variables associated with primary involvement of the lung appeared to be peripheral location, cavitation, mass size $>3 \mathrm{~cm}$ and mass-like consolidation (Fig. 2). Tumor localization involved the peripheral lobe and hilum, with hilar and mediastinal lymphadenopathy observed more in SPL (Fig. 2). All of these features associated with CT scan results were then used in a discriminant equation in an attempt to differentiate the diagnosis of PPL and SPL.

Overall accuracy of the established prediction equation was $92.1 \%$, with a false positive prediction in $0 \%$ for PPL and a false-positive prediction in $15.8 \%$ of SPL (Table II).

Prediction equation based on CT results. $\mathrm{L}=0.316-1.874$ (peripheral and hilar location) +2.211 (cavitation) +0.526 (mass $>3 \mathrm{~cm}$ ) -0.475 (hilar and mediastinal lymphadenopathy) +0.511 (consolidation) -0.010 (peripheral location). Features were encoded with $1=$ present and $0=$ absent. The coefficients were those obtained in the $\chi^{2}$ and Fisher's exact test analyses. Probability (belonging to PPL) $=1 /\left(1+\mathrm{e}^{-\mathrm{L}}\right)$.

Example: Patient 1, chest CT identified a peripheral mass $>3 \mathrm{~cm}$ with cavitation and hilar and mediastinal lymphadenopathy. The histological diagnosis was MALT of PPL. Patient $1: \mathrm{L}=0.316-1.874 \times 0+2.211 \times 1+0.526 \times 1-0.475 \times 1+0.511 \times$ $0-0.010 \times 1=2.568 . \mathrm{P}=1 /\left(1+\mathrm{e}^{-\mathrm{L}}\right)=0.929$.

This evaluation correctly predicted the diagnosis in $(19+19) / 38$ cases, with an overall accuracy of $92.1 \%$ (Table II).

\section{Discussion}

The present study analyzed the imaging features and clinical manifestations of PPL and SPL in an attempt to identify any differences and improve the understanding of their mechanisms and growth patterns, to ultimately contribute to the improved early diagnosis of PPL and SPL. Patients in the two groups may 


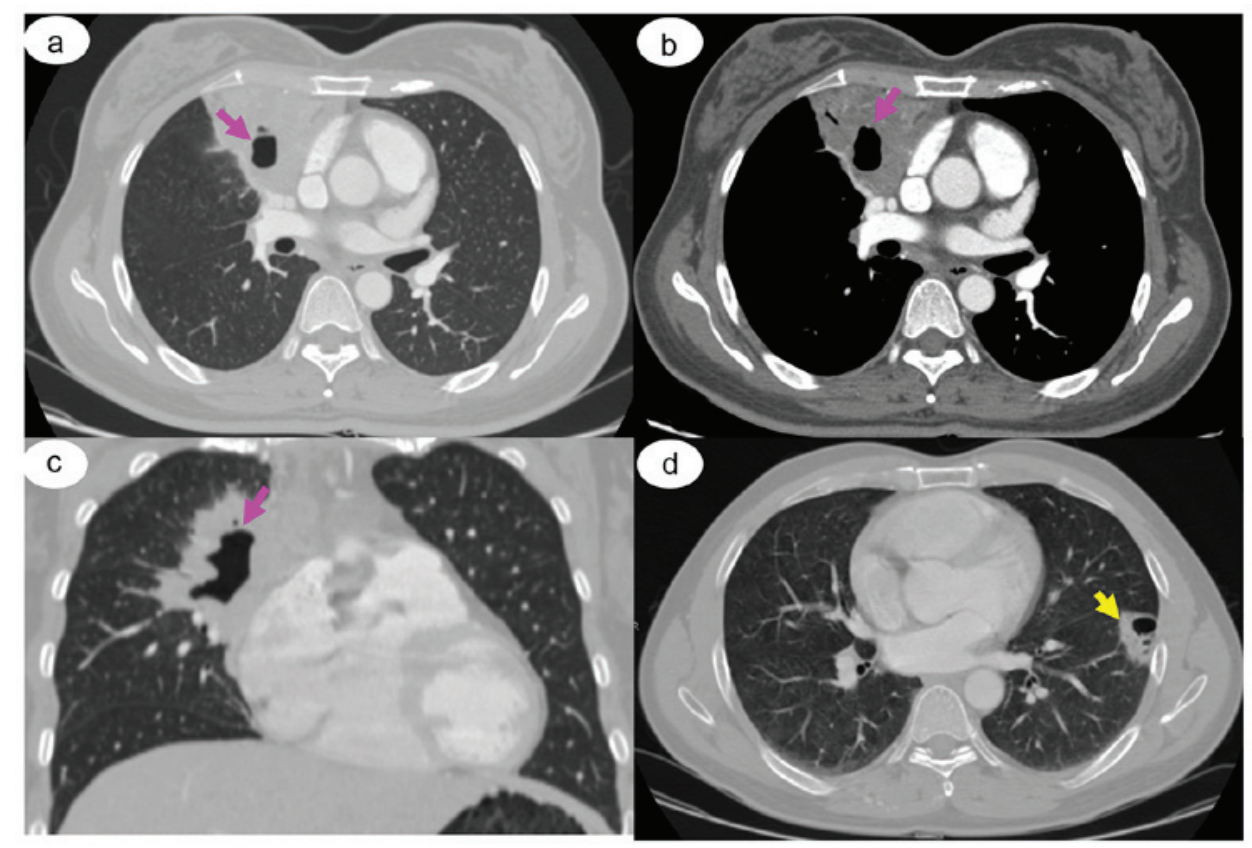

Figure 3. Representative CT images. Case of a 45-year-old woman with primary mucosa-associated lymphoid tissue lymphoma, confirmed by CT-guided transthoracic needle biopsy. Chest CT revealed a well-defined lesion with consolidation and cavitation in the right upper lobe of the lung (arrow) in (A) pulmonary window, (B) soft-tissue window and (C) coronal pulmonary window. (D) Case of a 43-year-old man with primary pulmonary Hodgkin's lymphoma, nodular sclerosis type, confirmed by bronchoscope biopsy. Chest CT revealed a peripherally located $4.5-\mathrm{cm}$ mass with cavitation in the left lower lobe of the lung (arrow).

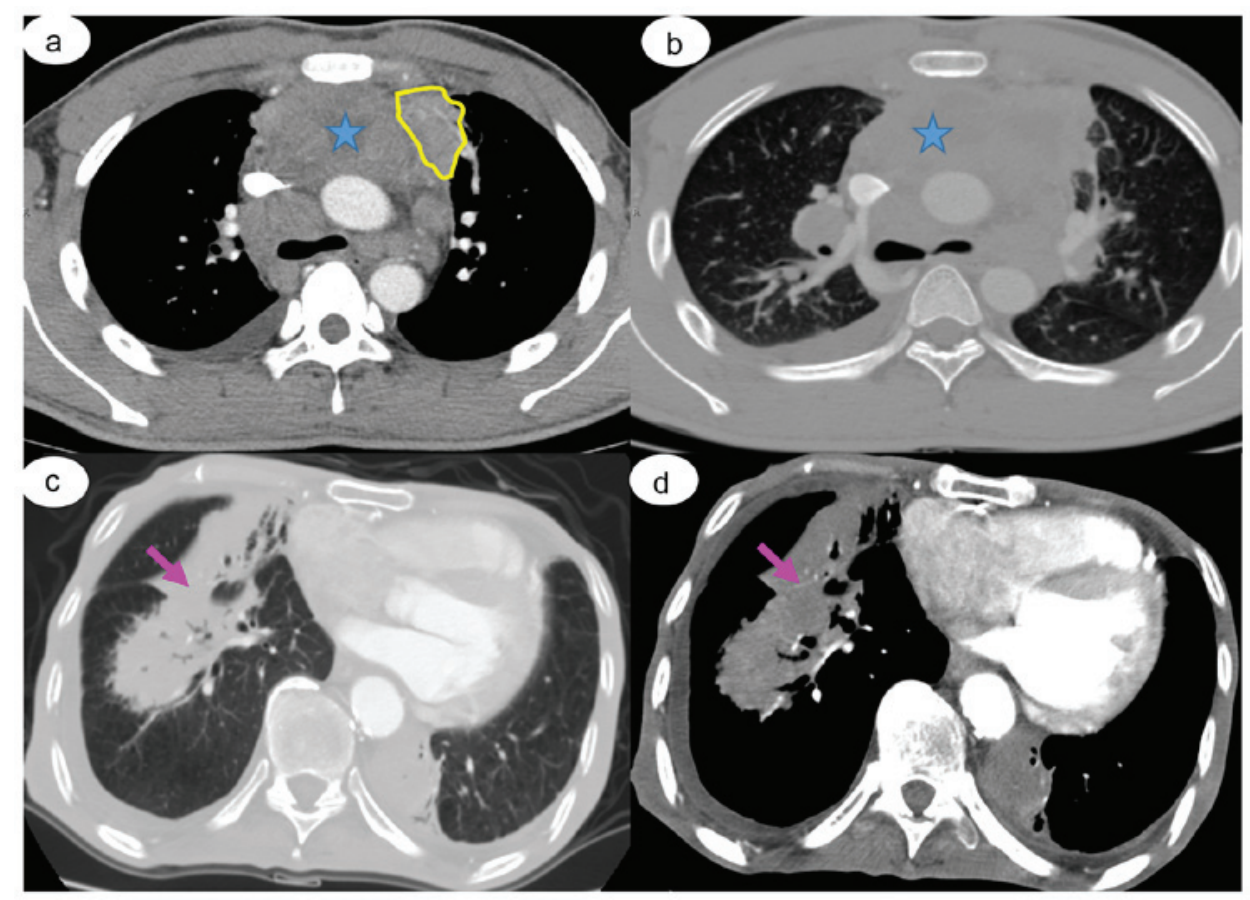

Figure 4. Representative CT images. Case of a 47-year-old man with secondary pulmonary diffuse large B lymphoma derived from the right axillary lymph nodes, confirmed by bronchoscopic biopsy. (A) Chest CT images revealed an ill-defined mass on the upper lobe of the left lung (yellow area) with multiple mediastinal lymphadenopathy. (B) Fusion and necrosis of the enlarged mediastinal lymph nodes (blue star) and a small amount of pleural effusion posteriorly was observed. Case of a 56-year-old man with secondary pulmonary non-Hodgkin's lymphoma, confirmed by bronchoscopic biopsy. Chest CT revealed bilateral diffuse lesions with irregular edges and an air bronchogram (arrow) in (C) pulmonary window and (D) soft-tissue window.

present with non-specific pulmonary symptoms or an unclear history of precedential lymphomas, which is consistent with previous studies $(19,20)$. The present study demonstrated that CT features may assist in the diagnosis of PPL and SPL with regard to tumor morphology and location, and lymph node involvement, but not with regard to the $\mathrm{SUV}_{\max }$ of PET/CT. 18F-FDG PET, which is based on functional metabolism rather than morphological mapping, serves an important role in the 
diagnosis and assessment of numerous types of malignancy, including lymphoma (21). However, this is not the case for the differentiation between PPL and SPL, as they share similar metabolism to the majority of lymphomas.

PPL is a malignant, monoclonal lymphoid proliferation within the lung parenchyma, presenting as a mass or nodules on CT images. MALT lymphoma is the major immunophenotype of PPL, accounting for 53\% of PPL in the present study. Bronchial mucosa-associated lymphoid tissue (BALT) is a type of MALT; a submucosal collection of lymphocytes (B and T cells) along the bronchial epithelium specializing in mucosal defense (5). BALT is absent from the lung in normal circumstances, developing in the bronchi only when there is chronic antigenic stimulation, and undergoing secondary lymphomatous transformation arising from marginal zone $\mathrm{B}$ cells, which may result in a peripheral mass (lower than the segmental bronchi) $(3,11,22)$. Cavitation and consolidation may occur subsequent to bronchi obstruction as the mass expands. Compared with PPL, SPL are malignant lymphoid growths disseminated from other sites, primarily through the mediastinal and hilar lymph nodes, to reach the lung parenchyma. Therefore, hilar and mediastinal lymphadenopathy was more commonly observed in SPL in the present study. Another possible explanation for the diffuse growth pattern of SPL may be that the majority of patients were diagnoses at a late stage, where the central and peripheral lung were affected.

Establishing radiological criteria for discrimination between these two types of lymphoma may be beneficial in guiding early diagnosis, evaluation and initiation of therapy $(13,23)$. This is important as therapeutic strategy and prognosis differ significantly between the two (19). PPL tends to present as an isolated mass or nodules that are treated with surgical resection alongside adjuvant chemotherapy or radiotherapy (24). However, SPL often presents with a disseminated pattern, referred to as advanced stage in the Ann Arbor classification (25), and polychemotherapy is recommended in the majority of cases (26). The prognosis for PPL is preferable to SPL, with SPL prognosis varying across distinct primary neoplasm immunophenotypes (27). An initial radiological diagnosis of SPL may suggest the use of further investigations, such as PET-CT or whole-body CT for overall evaluation and grading, which is recommended in the NCCN guidelines (12). Additionally, early detection of PPL may prevent unnecessary investigations, such as PET-CT in early-stage MALT lymphoma, considering that it is an indolent type with decreased incidence of distant metastasis compared with the disseminated pulmonary lymphoma $(2,5)$.

The results of the present study indicated that with the exception of MALT of NHL, other subtypes of lymphoma, including $\mathrm{T}$ cell and NK/T cell may also occur as primary neoplasms in the lung. The variability and similarity of lymphomas make an early diagnosis difficult (28). To the best of our knowledge, no precedential analysis focusing on the discriminative radiological features of PPL and SPL has previously been performed. The present study demonstrated that the observation of certain imaging features may assist in the accurate differential diagnosis of PPL and SPL. A mass or mass-like consolidation of homo-intense or marked heterogeneous enhancement with cavitation that favors a peripheral location was significantly associated with PPL, which is in line with the results of a previous study (29). SPL disseminated in a more diffuse pattern with hilar and mediastinal lymphadenopathy (1). A prediction equation based on features observed in CT results was adopted by means of discrimination analysis regarding 6 statistically significant features, with an accuracy of up to $92.1 \%$.

However, there are certain limitations to the present study. First, the sample size was limited and the cases were non-consecutive. Secondly, the study was retrospective. Thirdly, not all of the patients underwent PET-CT investigation, although all of them had undergone a whole-body CT scan and bone marrow aspiration. As a result, the present study focused more on the CT imaging. Finally, based on limited cases, the prediction equation derived from the canonical discriminate analysis warrants further verification from additional studies.

In conclusion, PPL and SPL present distinct features in CT images due to different molecular mechanism and growth patterns. The careful observation of CT images may facilitate the differential diagnosis of PPL and SPL with regard to tumor morphology and location, and lymph node involvement. This may improve the early diagnosis and guide further individualized investigation referrals for the patients, as well as aiding the early initiation of treatment strategies.

\section{Acknowledgements}

The present study was funded by the National Scientific Foundation of China (grant no. 81571664) and the Science and Technology Planning Project of Guangdong, China (grant no. 2014A020212244).

\section{References}

1. Carter BW, Wu CC, Khorashadi L, Godoy MC, de Groot PM, Abbott GF and Lichtenberger JP III: Multimodality imaging of cardiothoracic lymphoma. Eur J Radiol 83: 1470-1482, 2014.

2. Cardenas-Garcia J, Talwar A, Shah R and Fein A: Update in primary pulmonary lymphomas. Curr Opin Pulm Med 21: 333-337, 2015.

3. Borie R, Wislez M, Antoine M, Copie-Bergman C, Thieblemont C and Cadranel J: Pulmonary mucosa-associated lymphoid tissue lymphoma revisited. Eur Respir J 47: 1244-1260, 2016.

4. Miller DL and Allen MS: Rare pulmonary neoplasms. Mayo Clin Proc 68: 492-498, 1993.

5. Cadranel J, Wislez M and Antoine M: Primary pulmonary lymphoma. Eur Respir J 20: 750-762, 2002.

6. Kido T, Yatera K, Noguchi S, Sakurai Y, Nagata S, Kozaki M Tokuyama S, Ogoshi T, Kawanami T, Yoshii C, et al: Detection of MALT1 gene rearrangements in BAL fluid cells for the diagnosis of pulmonary mucosa-associated lymphoid tissue lymphoma. Chest 141: 176-182, 2012.

7. Wei S, Li X, Qiu X, Zhao H, Chen G, Chen J and Zhou Q: Primary lung lymphoma involving the superior vena cava. World J Surg Oncol 10: 131, 2012.

8. Zompi S, Couderc LJ, Cadranel J, Antoine M, Epardeau B, Fleury-Feith J,Popa N, Santoli F, Farcet JP and Delfau-Larue MH: Clonality analysis of alveolar B lymphocytes contributes to the diagnostic strategy in clinical suspicion of pulmonary lymphoma. Blood 103: 3208-3215, 2004.

9. Chua SC, Rozalli FI and O'Connor SR: Imaging features of primary extranodal lymphomas. Clin Radiol 64: 574-588, 2009.

10. Majid N, Kamal el B, Oncology B, Rachid A and Hassan IH: Primary pulmonary lymphoma: About five cases and literature review. Lung India 31: 53-55, 2014

11. Sirajuddin A, Raparia K, Lewis VA, Franks TJ, Dhand S, Galvin JR and White CS: Primary pulmonary lymphoid lesions: Radiologic and pathologic findings. Radiographics 36: 53-70, 2016.

12. National Comprehensive Cancer Network (NCCN) guideline in Hodgkin and non-Hodgkin lymphoma of oncology (version 2, 2015) (http://www.nccn.org). Accessed on April 26, 2016. 
13. $\mathrm{Xu} \mathrm{H}, \mathrm{Xu} \mathrm{K}$, Wang $\mathrm{R}$ and Liu X: Primary pulmonary diffuse large B-Cell lymphoma on FDG PET/CT-MRI and DWI. Medicine (Baltimore) 94: e1210, 2015.

14. Swerdlow SH, Campo E, Harris NL, et al (eds): WHO classification of tumors of haematopoietic and lymphoid tissues. IARC, Lyon, 2008.

15. Teoh EJ, McGowan DR, Bradley KM, Belcher E, Black E, Moore A, Sykes A and Gleeson FV: 18F-FDG PET/CT assessment of histopathologically confirmed mediastinal lymph nodes in non-small cell lung cancer using a penalised likelihood reconstruction. Eur Radiol 26: 4098-4106, 2016.

16. Wang L, Yin X, Wang F, Gu J, Lu L, Wu Q, Shen B and Li XF: The usefulness of combined diagnostic CT and (99m) Tc-octreotide somatostatin receptor SPECT/CT imaging on pulmonary nodule characterization in patients. Cancer Biother Radiopharm 28: 731-736, 2013.

17. Borie R, Wislez M, Thabut G, Antoine M, Rabbat A, Couderc LJ, Monnet I, Nunes H, Blanc FX, Mal H, et al: Clinical characteristics and prognostic factors of pulmonary MALT lymphoma. Eur Respir J 34: 1408-1416, 2009.

18. Ooi GC, Chim CS, Lie AK and Tsang KW: Computed tomography features of primary pulmonary non-Hodgkin's lymphoma. Clin Radiol 54: 438-443, 1999.

19. Niu X, Hu H, Gao J, Nie Y, Zhao W, Xu H, Bai X and Chen L: A clinical analysis of 40 cases of primary and secondary pulmonary lymphoma. Zhonghua Jie He He Hu Xi Za Zhi 37: 502-506, 2014 (In Chinese).

20. Xu J, Wu X and Reddy V: T Cell/Histiocyte-Rich large B cell lymphoma of the thymus: A diagnostic pitfall. Case Rep Hematol 2016: 2942594, 2016.

21. Wu F, Wang Z, Xing X, Yu M and Shi B: The value of 18F-FDG $\mathrm{PET} / \mathrm{CT}$ in diagnostic procedure of intravascular large B-Cell lymphoma presenting fever of unknown origin and pulmonary hypertension as an initial manifestation. Clin Nucl Med 41: 506-507, 2016.
22. Wislez M, Cadranel J, Antoine M, Milleron B, Bazot M, Mayaud C and Carette MF: Lymphoma of pulmonary mucosa-associated lymphoid tissue: CT scan findings and pathological correlations. Eur Respir J 14: 423-429, 1999.

23. Cheson BD, Horning SJ, Coiffier B, Shipp MA, Fisher RI, Connors JM, Lister TA, Vose J, Grillo-Lopez A, Hagenbeek A et al: Report of an international workshop to standardize response criteria for non-Hodgkin's lymphomas. NCI sponsored international working group. J Clin Oncol 17: 1244, 1999.

24. Parissis H: Forty years literature review of primary lung lymphoma. J Cardiothorac Surg 6: 23, 2011.

25. Takenaka $\mathrm{T}$ and Konda C: Hodgkin's disease staging classification and procedures, with special reference to Ann Arbor classification. Nihon Rinsho 41: 2469-2474, 1983 (In Japanese).

26. Fowler NH, Davis RE, Rawal S, Nastoupil L, Hagemeister FB, McLaughlin P, Kwak LW, Romaguera JE, Fanale MA, Fayad LE, et al: Safety and activity of lenalidomide and rituximab in untreated indolent lymphoma: An open-label, phase 2 trial. Lancet Oncol 15: 1311-1318, 2014.

27. Olszewski AJ and Castillo JJ: Survival of patients with marginal zone lymphoma: Analysis of the surveillance, epidemiology, and end results database. Cancer 119: 629-638, 2013.

28. Berkman N and Breuer R: Pulmonary involvement in lymphoma. Respir Med 87: 85-92, 1993.

29. Baccari-Ezzine S, Bouzaidi K, Chelbi E, Ali MB and Ghrairi H Unusual radiologic and histologic manifestations of primary pulmonary lymphoma. Asian Cardiovasc Thorac Ann 22: 362-364, 2014. 